\title{
Size of pelvic bone metastasis as a significant prognostic factor for metastatic prostate cancer patients
}

\section{Toyokazu Hayakawa}

Kitasato Daigaku Igakubu

Ken-ichi Tabata

Kitasato Daigaku Igakubu

Hideyasu Tsumura

Kitasato Daigaku Igakubu

\section{Shogo Kawakami}

Kitasato Daigaku Igakubu

\section{Takeo Katakura}

Kitasato Daigaku Iryo Eisei Gakubu

Masatoshi Hashimoto

Kitasato Daigaku Iryo Eisei Gakubu

\section{Yusuke Watanabe}

Kitasato Daigaku Iryo Eisei Gakubu

Masatsugu Iwamura

Kitasato Daigaku Igakubu

Tomoyuki Hasegawa

Kitasato Daigaku Igakubu

Hiromichi Ishiyama ( $\square$ hishiyam@kitasato-u.ac.jp )

Kitasato university https://orcid.org/0000-0001-7907-8422

\section{Research article}

Keywords: prostate cancer; radiomics; bone metastasis; computed tomography; pelvis

Posted Date: August 23rd, 2019

DOI: https://doi.org/10.21203/rs.2.13510/v1

License: (c) (i) This work is licensed under a Creative Commons Attribution 4.0 International License.

Read Full License 
Version of Record: A version of this preprint was published at Japanese Journal of Radiology on June 14th, 2020. See the published version at https://doi.org/10.1007/s11604-020-01004-5. 


\section{Abstract}

Background To investigate the potential prognostic value of image analysis of pelvic bone metastasis in newly diagnosed prostate cancer patients.Methods Data from 69 patients with both bone scintigraphy and pelvic CT images were selected for this analysis. Open source software (3D Slicer version 4.8.1.) was used for image analysis. Metastatic pelvic bone lesions were manually contoured, and radiomic features were extracted. As risk factors for overall survival (OS) and cause-specific survival (CSS), 105 radiomic features and clinical risk factors including age, initial prostate-specific antigen, Gleason score, TNM stage, lactate dehydrogenase $(\mathrm{LDH})$, hemoglobin $(\mathrm{Hb})$, alkaline phosphatase, extent of disease, visceral metastases, castration-resistant prostate cancer (CRPC), and number of systemic agents including abiraterone, enzalutamide, docetaxel and cabazitaxel which CRPC patients received were assessed by uni- and multivariate analyses. Results Median follow-up was 41 months (range, 0-157 months). Threeand 5 -year overall survival rates were $66.6 \%$ and $37.9 \%$, respectively. Three- and 5 -year cause-specific survival rates were $69.4 \%$ and $43.5 \%$, respectively. After multivariate analysis, $\mathrm{LDH}, \mathrm{Hb}$, and "maximum 2D diameter" defined as maximum tumor size in the axial plane were detected as risk factors for OS. Gleason sum, LDH, and maximum 2D diameter were detected as risk factors for CSS.Conclusion Maximum 2D diameter of pelvic bone metastasis was detected as a significant prognostic factor for metastatic prostate cancer patients.

\section{Background}

Patients with metastatic prostate cancer who receive systemic treatment survive an average of approximately 5 years ${ }^{1}$. This survival has barely changedeven in patients treated using current standards of chemotherapy ${ }^{2,3}$. However, survival in these patients is highly variable depending on the metastatic site and the burden of the metastatic tumor ${ }^{2,4}$. One of the reported prognostic factors for these patients is extent of disease (EOD), based on the number of bone metastasis counted on bone scintigraphy. Other widely used prognostic factors include categories based on not only the number of bone metastases, but also metastatic site and status of visceral metastasis, as used in the CHAARTED trial ${ }^{3}$ and STAMPEDE trial $^{2}$. In the current PSA era, the number of metastases in newly diagnosed patients is relatively small ${ }^{5}$ compared to that in the pre-PSA era ${ }^{6}$. According to our preliminary analysis, most metastatic prostate cancer patients had pelvic bone metastasis, and with metastases limited to the pelvic bone in a substantial number. Analysis focused on pelvic bone metastasis might thus be useful for predicting survival in recently diagnosed metastatic prostate cancer patients. This idea motivated the present study investigating the potential prognostic value of image analysis of pelvic bone metastasis.

\section{Methods}

This study was approved by the institutional review board of Kitasato University School of Medicine (B17-245). Medical records of 188 prostate cancer patients with positive results from bone scintigraphy diagnosed in our hospital between April 2003 and October 2014 were reviewed. The numbers of patients 
with metastasis to pelvic bone, vertebral bone, or other bone were 154 (81.9\%), 127 (67.6\%), and 120 (63.8\%), respectively. Thirty-three patients (17.6\%) showed metastasis limited to the pelvic bone.

We therefore focused on pelvic bone metastasis in this study, and data from 69 patients with both bone scintigraphy and pelvic CT images acquired in our hospital within the range of 1 month were selected for this analysis. Patient characteristics are shown in Table 1. Open source software (3D Slicer version 4.8.1I https://www.slicer.org/) was used for image analysis. Pelvic CT data were entered into the software and the metastatic lesion was contoured by one radiation oncologist (H. I.) using a segment editor module referring to bone scintigraphy of the same patient. Scalar volume was resampled into a size of $3 \mathrm{~mm} \times 3$ $\mathrm{mm} \times 3 \mathrm{~mm}$. Radiomic features were extracted from contoured images using a radiomics module. As risk factors for overall survival (OS) and cause-specific survival (CSS), 105 radiomic features including shapebased $(n=13)$, gray-level dependence matrix $(n=14)$, gray-level co-occurrence matrix $(n=23)$, first-order statistics $(n=18)$, gray-level run length matrix $(n=16)$, gray-level size zone matrix $(n=16)$, and neighboring gray tone difference matrix $(n=5)$ were assessed by univariate analysis. Significant variables on univariate analysis were included in the multivariate analysis.

As clinical risk factors for OS and CSS, age, initial PSA, Gleason score, TNM stage, lactate dehydrogenase $(\mathrm{LDH})$, hemoglobin $(\mathrm{Hb})$, alkaline phosphatase (ALP), and EOD visceral metastases, CRPC, and number of systemic agents including abiraterone, enzalutamide, docetaxel and cabazitaxel which CRPC patients received were assessed by univariate analysis. For clinical risk factors, $p$ value $<0.05$ was considered as significant. For radiomic features, $p$ value $<0.00047$ was considered to denote statistical significance according to Bonferroni correction for 0.05 decision threshold. Significant radiomic features from multivariate analysis and significant clinical factors from univariate analysis were included in the final multivariate analysis. All univariate and multivariate analysis were done by using Cox proportional hazard models.

\section{Results}

Median follow-up time for the 69 patients was 41 months (range, 0-157 months). During follow-up, 43 patients died. Of these, 37 patients died of prostate cancer, and the other patients died of heart failure ( $\mathrm{n}$ $=1)$, brain hemorrhage $(n=1)$, aspiration pneumonia $(n=1)$, and unknown causes $(n=3)$. Three and 5year OS rates were $66.6 \%$ and $37.9 \%$, respectively. Three and 5 -year CSS rates were $69.4 \%$ and $43.5 \%$, respectively.

Table 2 shows the results of uni- and multivariate analyses of radiomic features. Only shape-based features were detected as risk factors for OS, and "maximum 2D diameter", defined as the largest size of the tumor surface in the axial plane, was detected as a risk factor for OS after multivariate analysis. None of the risk factors for CSS were detected after uni- and multivariate analyses

Tables 3 and 4 show the results of uni- and multivariate analyses of clinical parameters and maximum 2D diameter. $\mathrm{LDH}, \mathrm{Hb}$, and maximum 2D diameter were detected as risk factors for OS after multivariate 
analysis. Total Gleason score, LDH, and maximum 2D diameter were detected as risk factors for CSS after multivariate analysis, although EOD was not.

Figure 1 shows the relationships between OS time and maximum 2D diameter. A significant relationship was evident between the two parameters.

\section{Discussion}

In 1940, Batson et al. reported a very abundant venous plexus around the pelvic bone and vertebrae, as revealed by intravenous injection of contrast material via the dorsal penile vein ${ }^{7}$. This plexus, now called Batson's plexus, suggests that much of the venous flow, and thus prostate cancer cells, from the prostate gland would run to the pelvic bone and vertebrae. Bone is definitely the most common site of distant metastasis from prostate cancer, and reportedly $86 \%$ of prostate primary tumors show only bone metastases $^{8}$.

Our study revealed that the size of a pelvic bone metastasis is a significant risk factor for CSS and OS after multivariate analysis that included EOD and other clinical risk factors. A large variation in survival time is seen among metastatic prostate cancer patients, and many of patients with small pelvic bone metastasis experience relatively long survival (Figure 1). These results suggest that we need to take the pelvic bone separately from other sites of bone metastasis. According to Batson's suggestion, pelvic and vertebral bones might represent a direct drainage system for cancer cells, in the manner of the lymph node system for other types of cancer. Local treatment such as radiotherapy rather than systemic chemotherapy might thus benefit patients with small pelvic metastasis.

EOD is one of the standard predictive factors for patients with metastatic prostate cancer. However, the significance of EOD disappeared after multivariate analysis in our study, although maximum size of the pelvic bone metastasis remained as a significant factor. This result suggests that a simple measurement of pelvic bone metastasis might be better than EOD for predicting survival, and is useful in routine practice even in hospitals without bone scintigraphy.

Recent development of image analysis using radiomic software has helped in the acquisition of additional information from traditional imaging modalities such as $\mathrm{CT}$, and this information could be available via freely distributed software such as 3D Slicer. As the value of the 2D diameter seems relatively stable among various images, the robustness of the radiomic feature was not assessed in this study. Intriguingly, among 105 radiomic features, simple measurement of the size of the pelvic bone metastasis was sufficient to predict survival time.

Our study has several limitations that must be considered: 1) the retrospective manner of the data collection might have introduced some biases; and 2) the same examiner contoured metastatic lesions based on CT and bone scintigraphy, and some variability might be introduced if contouring were performed by other examiners. Further prospective assessments are warranted. 


\section{Conclusion}

Maximum 2D diameter of pelvic bone metastasis was detected as a significant prognostic factor for metastatic prostate cancer patients.

\section{Abbreviations}

OS: overall survival; CSS: cause-specific survival; LDH: lactate dehydrogenase; Hb: hemoglobin; CRPC: castration-resistant prostate cancer; EOD: extent of disease; ALP: alkaline phosphatase

\section{Declarations}

\section{Ethics approval and consent to participate}

This study was approved by the institutional review board of Kitasato University School of Medicine (B17-245). No consent was required as this study used anonymized registry data.

\section{Consent for publication}

Not applicable.

\section{Availability of data and materials}

The datasets generated and/or analyzed during the current study are not publicly available.

\section{Competing interests}

The authors declare that they have no competing interests

\section{Funding}

Not applicable.

\section{Authors' contributions}

TH and $\mathrm{HI}$ drafted the manuscript. TH, KT, HT, SK, TK, MH, YW and HI participated in the design of the study. TH and $\mathrm{HI}$ performed the statistical analysis. MI and TH supervised the study. TH, KT, HT, SK, TK, $\mathrm{MH}, \mathrm{YW}$ and $\mathrm{HI}$ collected medical information about this study. 


\section{Acknowledgment}

Not applicable.

\section{References}

1.Pound CR, Partin AW, Eisenberger MA, Chan DW, Pearson JD, Walsh PC. Natural history of progression after PSA elevation following radical prostatectomy. JAMA. 1999;281(17):1591-1597.

2.Parker CC, James ND, Brawley CD, et al. Radiotherapy to the primary tumour for newly diagnosed, metastatic prostate cancer (STAMPEDE): a randomised controlled phase 3 trial. Lancet. 2018.

3.Sweeney CJ, Chen YH, Carducci M, et al. Chemohormonal Therapy in Metastatic Hormone-Sensitive Prostate Cancer. N Engl J Med. 2015;373(8):737-746.

4.Gandaglia G, Karakiewicz PI, Briganti A, et al. Impact of the Site of Metastases on Survival in Patients with Metastatic Prostate Cancer. Eur Urol. 2015;68(2):325-334.

5.Koizumi M, Motegi K, Koyama M, Terauchi T, Yuasa T, Yonese J. Diagnostic performance of a computer-assisted diagnosis system for bone scintigraphy of newly developed skeletal metastasis in prostate cancer patients: search for low-sensitivity subgroups. Ann Nucl Med. 2017;31(7):521-528.

6.Soloway MS, Hardeman SW, Hickey D, et al. Stratification of patients with metastatic prostate cancer based on extent of disease on initial bone scan. Cancer. 1988;61(1):195-202.

7.Batson OV. The Function of the Vertebral Veins and Their Role in the Spread of Metastases. Ann Surg. 1940;112(1):138-149.

8. Hess KR, Varadhachary GR, Taylor SH, et al. Metastatic patterns in adenocarcinoma. Cancer. 2006;106(7):1624-1633.

\section{Table 1}

Due to technical limitations, Table 1 is only available as a download in the supplemental files section.

\section{Figures}




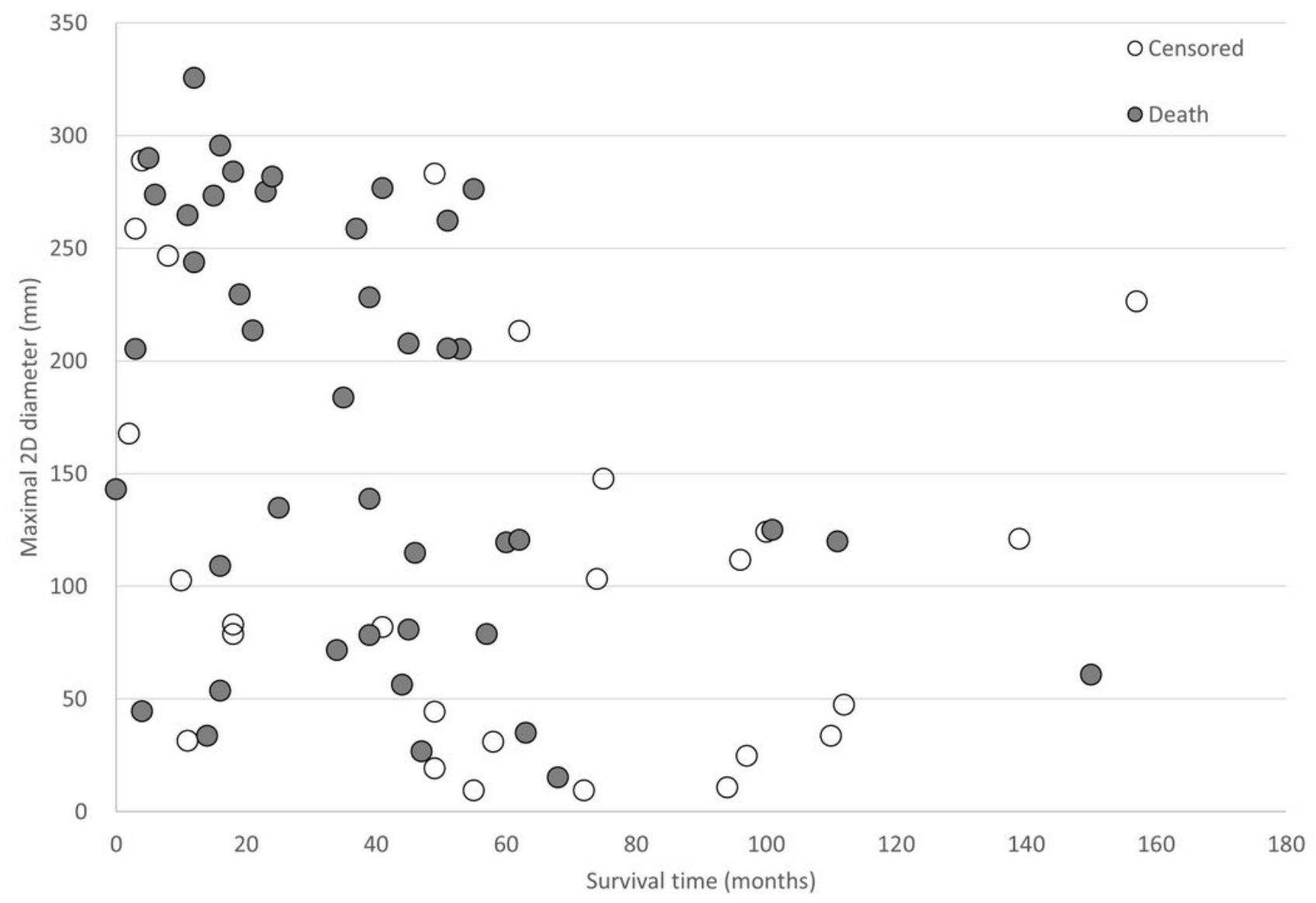

Figure 1

Relationships between maximum 2D diameter at the pelvic bone metastasis and survival time in patients with newly diagnosed metastatic prostate cancer.

\section{Supplementary Files}

This is a list of supplementary files associated with this preprint. Click to download.

- supplement1.tif

- supplement2.tif

- supplement3.tif

- supplement4.tif 